DOI: $10.52837 / 27382702-2021-34.1-158$

\title{
TWO ARMENIAN PERSONAL NAMES WITH ŠAH ‘KING”*
}

\author{
Hrach Martirosyan 1
}

To the memory of Samvel Karapetyan

\begin{abstract}
There are a great number of Armenian compound personal names with the element šah 'king' of Iranian origin (Middle Persian and New Persian šāh 'king'). It occurs: (1) in both masculine and feminine names; (2) with both native Armenian and foreign components; (3) either as the first or the second component; (4) often in doublet forms with a reversed order of the components. For instance: masculine Šah-amir and Amir-šah, Šah-paron and Paron-šah, Vahram-šah; feminine: Šah-xat'un and Xat'unšah, Šah-tikin. Also note masc. Šah-aziz vs. fem. Aziz-šah, masc. Sult'an-šah vs. fem. Šah-sult'an, masc. Melik'-šah vs. fem. Šah-melē/ik'² (the latter is sometimes masculine, cf. Middle Persian > Syriac Šāh-malīk, also masculine³).

This paper aims to interpret two hapax legomena in which the component šah became synchronically unanalyzable due to phonological changes. In one of them, šah is the second member of the name (gen. Artamšin < *Artam-šah/y-in), whereas in the other it is the first one (Šašt' $i<$ * Šah-st' $i$ 'Šah-Lady').
\end{abstract}

Keywords: Middle Persian, New Persian, Armenian, Šah-amir, Vahram-šah, Sult'anšah, Šah-paron, Melik'-šah.

1. Artamšah, gen.-dat. Artamši-n

In an inscription from Horromos (Širak), 1266 CE, one finds dative Artamšin as the name of a relative of Mamukst $i$ (for the latter, see $\S 2$ ) ${ }^{4}$. The

\footnotetext{
* The article was submitted on May 21, 2021: The article was reviewed on June 29, 2021.

1 The research on which this work is based has taken place in the framework of the Project no. P 27029-G23 of the Austrian Science Fund (FWF) carried out at the Institute of Iranian Studies at the Austrian Academy of Sciences.

2 See AčaṙAnjn s.vv.

${ }^{3}$ Gignoux / Jullien / Jullien 2009: 129 Nr. 395.

${ }^{4}$ Karapetyan 2015: 466; see also Ališan 1881: 26b; Kostaneanc' 1913: 109.
} 
nominative of this personal name (henceforth: PN) is not attested. Three suggestions have been made for the nominative form:

- *Artamuš (Ališan 1881: 182a);

- *Artamšah, with šah 'king’ (AčaṙAnjn 1, 1942: 304);

- *Artamiš (Karapetyan 2015: 466).

Below I will argue that Ačaryan's reconstruction is the correct one.

This name, in my opinion, is based on ${ }^{*}$ Artam from Old Iranian ${ }^{*} \mathrm{R}$ 。 tā ${ }^{\top}$ ma-: Elam. Ir-da-ma, Aram. 'rtm, Babyl. Ar-ta-am-ma-' m., Gr. (Egypt.) 'A 'impetuosity, strength, power', thus: 'having power of/from R。ta' (cf. Av. ama-, Skt. áma- 'id.' 'power'), ${ }^{5}$ compare Arm. Aršam from the Iranian m. PN *Ršāma- < *rša-ama- 'having power of a man/hero' with *ršan- 'man, hero', cf.

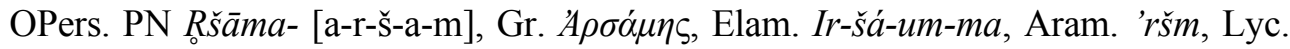
Arssãma-, Babyl. Ar-šá-am-ma-', Ar-šá-am-mu, Ar-šá-am ${ }^{6}$.

The auslaut of the Armenian name needs an explanation. One might start with Ačaryan's *Artam-šah, with the well-known Iranian word for 'king' (Middle Persian and New Persian šāh 'king'), which is also found as a PN not only in Iranian languages but also in Arabic, Armenian, etc ${ }^{7}$. The intervocalic $h$ - has been lost in the dative, which is the only attested form; thus: *Artamš(əh)-in. This is confirmed by several clear examples such as Šahnšayi, Šahanši and Šayinši (genitives of Šahanšah or Šaha/inšay) and Asuši (genitive of Asušah $)^{8}$. In a colophon from Van, $1460 \mathrm{CE}^{9}$ one finds f. PN Xat'unš-in

\footnotetext{
5 Alternatively, one assumes a two-stem short name ${ }^{*} R$ t $t a-m-a$ - to ${ }^{*} R$ 。 ta-manah- ' $R$ 。 taminded'. For the forms, see Schmitt 1972: 87-89, 2002: 83 fn. 6, 2011: 101-102, 104; Huyse 1990: 37 Nr. 34; Tavernier 2007: 297; Zadok 2009: 102 Nr. 65; Schmitt / Vittmann 2013: 46-48. For the *ama-component, see also Martirosyan 2019: 332a on Amatuni.

${ }^{6}$ Hübschmann 1897: 27; AčaṙAnjn 1, 1942: 294; Brandenstein / Mayrhofer 1964: 106; Mayrhofer 1977: 43, 1979: 12; Benveniste 1966: 85; Schmitt 1978: 23-25, 1982a: 375, 1982b: 18, 1998: 180, 2006: 77-80; Ayvazian-Terzian 1999-2000: 414; Zadok 2009: 86-87, 98.

7 Justi 1895: 271ff.; Hübschmann 1897: 58f.; AčaṙAnjn 4, 1948: 102.

8 See Avagyan 1973: 298, 376-377.

${ }^{9}$ See HayJeṙHiš XV.2, 1958: 146.
} 
and m. PN Sult'anš-in, which are the genitive-dative forms of Xat'unšah and Sult'anšah frequently spelled also Xat'unšay and Sult'anšay ${ }^{10}$.

Decisive proof is provided in the very same inscription of Horomos, in which Artamšin is followed by Varhamšin, which should be interpreted exactly in the same way, viz. genitive-dative of Varhamšah.

One may wonder if Artumše attested in a post-1602 colophon and reflecting Artumšah ${ }^{11}$ belongs here too. Note that from the 12th century the element Šah frequently yields Šay (cf. above) ${ }^{12}$, thus šah- $n$, with def. article $-n$, and becomes * šay- $n>$ šè- $n$. Compare Amiršse $n<*$ Amir-šah/y- $n$ and Surmanšēe $n$ $<$ *Surman-šah/y-n in a colophon from the church called Tivriku Surb Astuacacin, $1694 \mathrm{CE}^{13}$.

We may thus safely conclude that the nominative of Artamš-in is indeed Artamšah, consisting of *Artam and šah 'king'.

\section{2. Šašt'i}

This is a f. PN only found in a colophon from the Kołbay monastery, 1289 CE, ${ }^{14}$ as the name of a sister of Dawit' and priest ( $k^{\prime}$ ahanay) Vardan. It is not recorded in AčaṙAnjn.

I interpret this name as consisting of šah 'king' and *st'i 'Lady'15. The latter member reflects Arab. sit(t)i 'My lady, lady' (cf. also NPers. sitti 'My lady, lady ${ }^{\prime 16}$ ) and is found in Armenian both independently (f. PN St 'i or Sz/it ' $i$ ) and as the second component of such feminine compound names as:

Gohar-s(i)t 'i, with gohar 'gem', thus 'Gohar/Gem-Lady';

\footnotetext{
${ }^{10}$ AčaṙAnjn 2, 1944: 450 and 4, 1948: 583-585.

${ }^{11}$ AčaṙAnjn 1, 1942: 319.

12 Gyulbudałyan 1973: 114; Avagyan 1973: 298.

${ }^{13}$ Srvandztyants T'A 1, 1879: 220; AčaṙAnjn 4, 1948: 590

14 HayJerrHiš XIII, 1984: 621; Harutyunyan 2018: 210-211. I am indebted to Khačik Harut'yunyan for this information.

${ }^{15}$ Martirosyan apud Harutyunyan 2018: 210 with fn. 56.

${ }^{16}$ See Steingass 1892: 657a.
} 
Mam-st ' $i$, with mam 'mother', thus 'Mother-Lady' (also Mamuk-st'i, with mamuk' 'grandmother' $)^{17}$.

These Armenian f. compound names are comparable with Gohar-tikin and Mama-tikin respectively, both consisting of the same first members gohar 'gem' and mama 'mother', and the native Armenian word for 'lady', namely tikin $^{18}$.

Thus, the f. PN Šašt $i$ can be interpreted as *Šah $+s t^{\prime} i$ 'Šah-Lady' (with

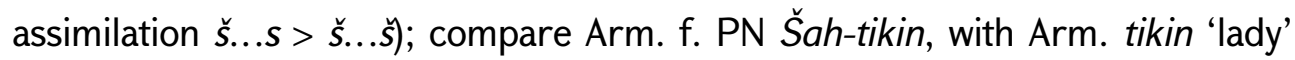
as the second member of this compound name ${ }^{19}$.

The striking parallelism between all these names can be observed in the table below.

\begin{tabular}{|l|l|l|}
\hline & + tikin 'lady' & + st $^{\text {'i }}$ 'lady' \\
\hline gohar 'gem' + & Gohar-tikin & Gohar-s(i)t'i \\
\hline mam(a) 'mother' + & Mama-tikin & Mam(uk)-st'i \\
\hline šah 'king' + & Šah-tikin & $*$ Šah-st'i \\
\hline
\end{tabular}

\section{BIBLIOGRAPHY}

AčaṙAnjn 1-5 - Ačaŕya H., Hayoc' anjnanunneri baraaran (Dictionary of Armenian personal names), h. 1, 1942; h. 2, 1944; h. 3, 1946; h. 4, 1948; h. 5, 1962. Yerevan: (In Armenian).

Ališan Ğ. 1881: Širak: tełagrut'iwn patkerac'oyc‘. Venice: S. Lazar. (In Armenian).

Avagyan S. A. 1973: Vimakan arjanagrut'yunneri hnč'yunabanut'yun (X-XIV dd.). Yerevan: (In Armenian).

Avagyan S. A. 1978: Vimakan arjanagrut'yunneri barak'nnut'yun. Yerevan: University Press.

Avagyan S. A. 1986: Vimagrakan prptumner. Yerevan: (In Armenian).

17 Also note f. PN Mehrast'i, attested in a 13th century colophon (zhanguc'eal mayrn im zMehrast' in ew zk'oyrn im zAłuan, see HayjeriHiš XIII, 1984: 887) and probably consisting of *MiOra- > *Mehr(a)-component and St' $i$ 'Lady'.

18 AčaṙAnjn 1, 1942: 486; 3, 1946: 186-187; 4, 1948: 491-492, cf. 473; Avagyan 1978: $291-$ 294, 1986: 194-199. For Mamukst'i, see also $§ 1$.

${ }^{19}$ See AčaṙAnjn 4, 1948: 131. 
Ayvazian-Terzian M.1999-2000: Persian proper names. In: Iran \& Caucasus 3-4: 413-414.

Benveniste É. 1966: Titres et noms propres en iranien ancien. Paris: Librairie C. Klincksieck. (Travaux de I'Institut d'Études Iraniennes de l'Université de Paris; 1).

Brandenstein W. \& Mayrhofer M. 1964: Handbuch des Altpersischen. Wiesbaden: Otto Harrassowitz.

Gignoux Ph., Christelle J., Florence J. 2009: Noms propres syriaques d'origine iranienne. In: Iranisches Personennamenbuch (hrsg. von Rüdiger Schmitt, Heiner Eichner, Bert G. Fragner und Velizar Sadovski). Band VII, Faszikel 5. Wien: Verlag der Österreichischen Akademie der Wissenschaften.

Gyulbudağyan S. V 1973: Hayereni uğğagrut'yan patmut'yun (History of Armenian orthography). Yerevan: (In Armenian).

Harutyunyan Kh. 2018: Anjnanunnerə hayeren jeṙagreri hišatakarannerum: 1. Norahayt anjnanunner ŽA-ŽG (XI-XIII) dareri hišatakaranneric ' (Personal Names in Armenian Colophons: 1 Newly Discovered Personal Names in the Colophons of $11^{\text {th }}$ $13^{\text {th }}$ Century Armenian Manuscripts), Banber Matenadarani (Yerevan) 25: 187-21 7 (In Armenian).

HayJerHiš 1950-88: - Hayeren jeṙagreri hišatakaranner: V-XVII dd. (ed. by L. Khač‘ikyan, A. S. Mat'evosyan et al.). Yerevan: Academy Press (in progress).

Hu“bschmann H. 1897: Armenische Grammatik. 1. Theil: Armenische Etymologie. Leipzig: Breitkopf \& Ha“rtel [The first part of the book (pp. 1-280) was first published in 1895].

Huyse Ph.1990: Iranische Namen in den griechischen Dokumenten Ägyptens. In: Iranisches Personennamenbuch (hrsg. von Manfred Mayrhofer und Rüdiger Schmitt). Band V, Faszikel 6a. Wien: Verlag der Österreichischen Akademie der Wissenschaften.

Justi F. 1895: Iranisches Namenbuch. Marburg. Reprinted in 1963, Hildesheim: Georg Olms Verlagsbuchhandlung; in 2004, Teheran: Asatir (mit einem persischen Vorwort von Dr. Ali Ashraf Sadeghi).

Karapetyan S. 2015: The epigraphy of Horomos: a historical survey of research. In: Hoŕomos monastery: art and history (ed. by Edda Vardanyan). Paris: Collège de France, CNRS (Centre de recherche d'histoire et civilisation de Byzance; Monographies 50): 391-489. 
Kostaneants K.1913: Vimakan taregir: c'uc'ak žołovacoy ardzanagrut'eanc' hayoc'. S. Peterburg: Tparan Gitut'eanc' čemarani Kayserakani. (In Armenian)

Martirosyan H. 2019: The Armenian patronymic Arcruni. In: Over the mountains and far away: studies in Near Eastern history and archaeology presented to Mirjo Salvini on the occasion of his 80th birthday (ed. by Pavel S. Avetisyan, Roberto Dan and Yervand H. Grekyan). Oxford: Archaeopress Publishing Ltd.: 331-336.

Mayrhofer M. 1977: Zum Namengut des Avesta. Wien: Verlag der Österreichischen Akademie der Wissenschaften. (Österreichische Akademie der Wissenschaften. Philosophisch-historische Klasse. Sitzungsberichte, 308.5. Vero“ffentlichungen der iranischen Kommission, 3).

Mayrhofer M. 1979: Die altpersischen Namen. In: Iranisches Personennamenbuch (hrsg. von Manfred Mayrhofer). Band I: Die altiranischen Namen, Faszikel 2 (und Faszikel 3: Indices). Wien: Verlag der Österreichischen Akademie der Wissenschaften. (Österreichische Akademie der Wissenschaften. Philosophischhistorische Klasse. Sonderpublikation der iranischen Kommission).

Schmitt R. 1972: Persepolitanisches. III. In: ZVS 86.1: 82-92.

Schmitt R. 1978: Die Iranier-Namen bei Aischylos (Iranica Graeca Vetustiora. I). Wien: Verlag der Österreichischen Akademie der Wissenschaften. (Österreichische Akademie der Wissenschaften. Philosophisch-historische Klasse. Sitzungsberichte, 337. Vero “ffentlichungen der Iranischen Kommission; 6).

Schmitt R. 1982a: Iranische Wörter und Namen im Lykischen. In: Serta indogermanica: Festschrift für Günter Neumann zum 60. Geburtstag (hrsg. von Johann Tischler). Innsbruck: Institut fu“ $r$ Sprachwissenschaft der Universita"t Innsbruck. (Innsbrucker Beitra“"ge zur Sprachwissenschaft; 40): 373-388.

Schmitt R. 1982b: Iranische Namen in den indogermanischen Sprachen Kleinasiens (Lykisch, Lydisch, Phrygisch). In: Iranisches Personennamenbuch (hrsg. von Manfred Mayrhofer und Rüdiger Schmitt). Band V, Faszikel 4. Wien: Verlag der Österreichischen Akademie der Wissenschaften.

Schmitt R. 1998: Parthische Sprach- und Namenüberlieferung aus arsakidischer Zeit. In: Das Partherreich und seine Zeugnisse (The Arsacid Empire: sources and documentation): Beiträge des internationalen Colloquiums, Eutin, 27. - 30. Juni 1996 (ed. by Josef Wiesehöfer). Stuttgart: Franz Steiner Verlag: 163-204.

Schmitt R. 2002: Die iranischen und Iranier-Namen in den Schriften Xenophons. (Iranica Graeca Vetustiora. II). Wien: Verlag der Österreichischen Akademie der Wissenschaften. (Österreichische Akademie der Wissenschaften. Philosophisch- 
historische Klasse. Sitzungsberichte, 692. Vero“ffentlichungen der Kommission für Iranistik, 29).

Schmitt R. 2006: Iranische anthroponyme in den erhaltenen Resten von Ktesias' Werk (Iranica Graeca Vetustiora. III). Wien: Verlag der Österreichischen Akademie der Wissenschaften. (Österreichische Akademie der Wissenschaften. Philosophisch-historische Klasse. Sitzungsberichte, 736. Vero“ffentlichungen zur Iranistik, 33).

Schmitt R. 2011: Iranische Personennamen in der griechischen Literatur vor Alexander d. Gr. In: Iranisches Personennamenbuch (hrsg. von Rüdiger Schmitt, Heiner Eichner, Bert G. Fragner und Velizar Sadovski). Band V, Faszikel 5A. Wien: Verlag der Österreichischen Akademie der Wissenschaften.

Schmitt R., Vittmann G. 2013: Iranische Namen in ägyptischer Nebenüberlieferung. In: Iranisches Personennamenbuch (hrsg. von Rüdiger Schmitt, Heiner Eichner, Bert G. Fragner und Velizar Sadovski). Band VIII. Wien: Verlag der Österreichischen Akademie der Wissenschaften. (Iranische Onomastik 13; Österreichische Akademie der Wissenschaften: Philosophisch-historische Klasse. Sitzungsberichte; 842).

Srvandztyants G. 1879-84: (T‘A 1-2), T'oros Ałbar Hayastani čambord (2 vols.). Pōlis: Tntesean / Pałtatlean (Aramean).

Steingass F. 1892: A comprehensive Persian-English dictionary: including the Arabic words and phrases to be met with in Persian literature: being Johnson and Richardson's Persian, Arabic and English dictionary: revised, enlarged and entirely reconstructed (reprinted in 1977). London: Routledge \& Kegan Paul. (= 2000, New Delhi: Munshiram Manoharlal Publishers).

Tavernier J. 2007: Iranica in the Achaemenid period (ca. 550-330 B.C.): lexicon of Old Iranian proper names and loanwords, attested in non-Iranian texts. Leuven, Paris, Dudley MA: Peeters. (Orientalia Lovaniensia analecta; 158).

Zadok R. 2009: Iranische Personennamen in der neu- und spätbabylonischen Nebenüberlieferung. In: Iranisches Personennamenbuch (hrsg. von Rüdiger Schmitt, Heiner Eichner, Bert G. Fragner und Velizar Sadovski). Band VII, Faszikel 1B. Wien: Verlag der Österreichischen Akademie der Wissenschaften.

Hrach Martirosyan

Leiden (Netherlands) hrch.martirosyan@gmail.com 


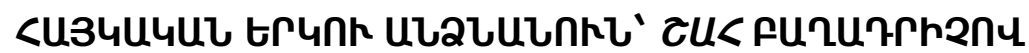

\section{<nus Uunuphnnujur}

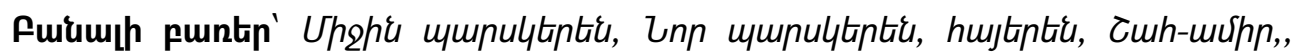

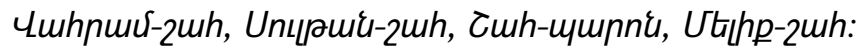

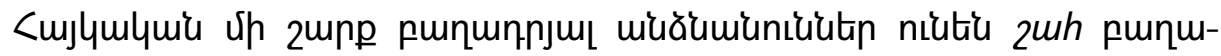

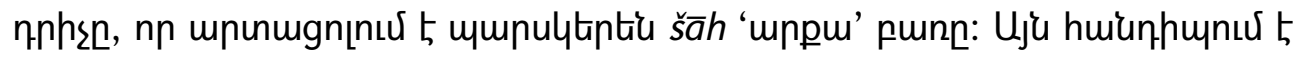

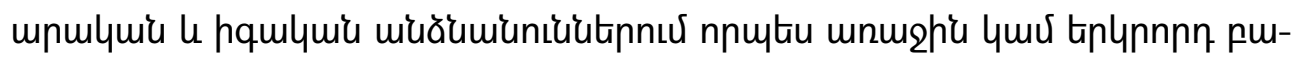

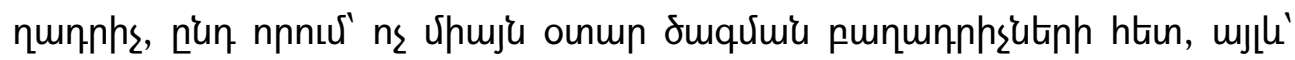

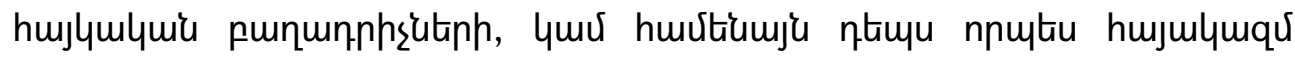

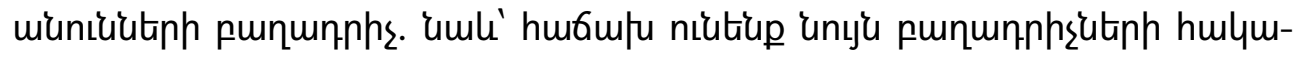

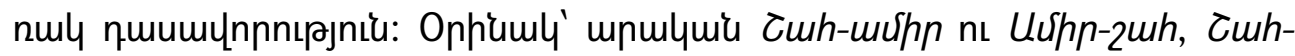

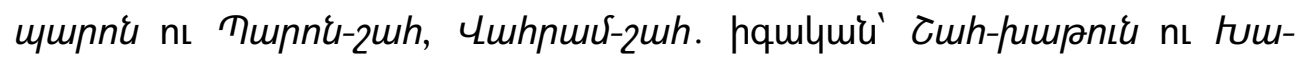

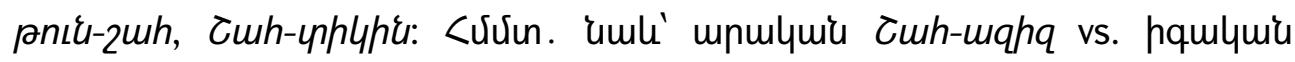

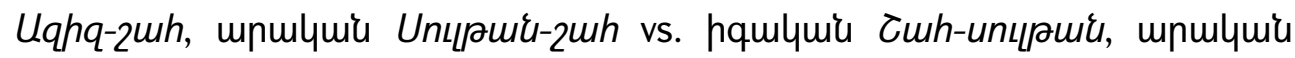

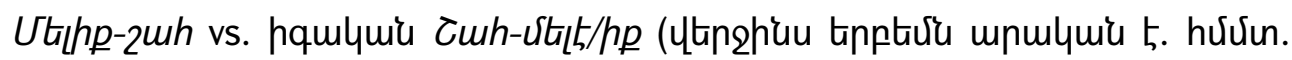

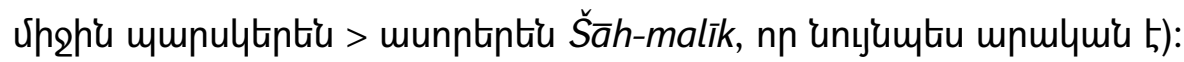

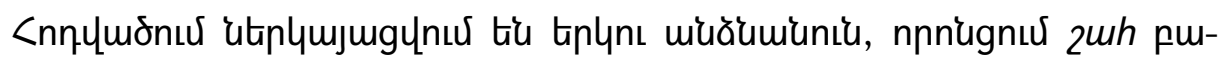

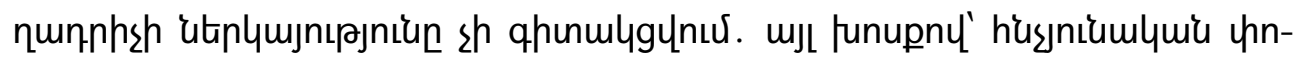

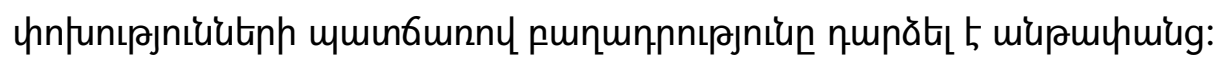

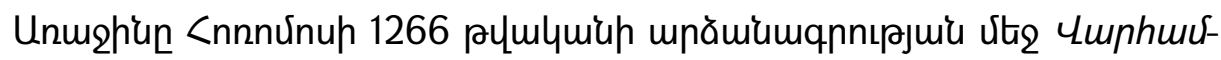

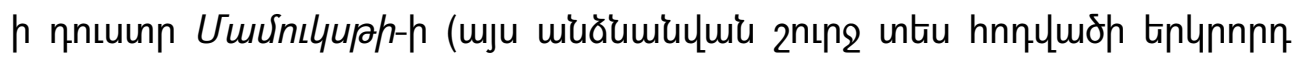

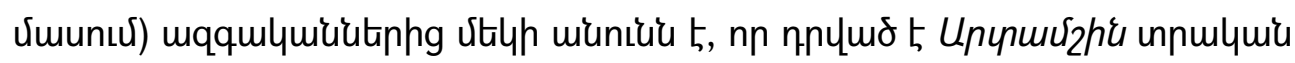

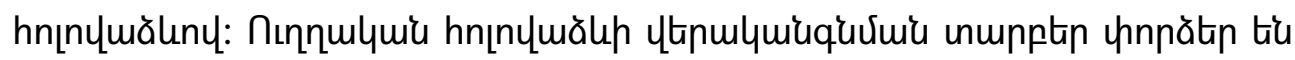

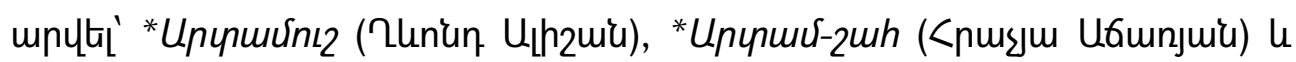

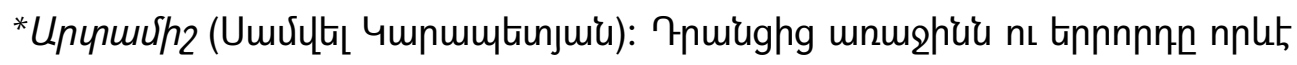

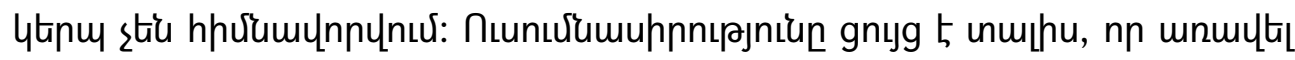

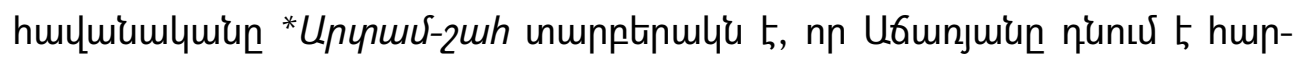

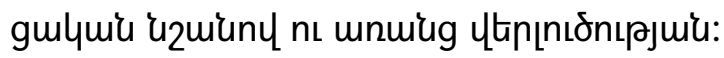

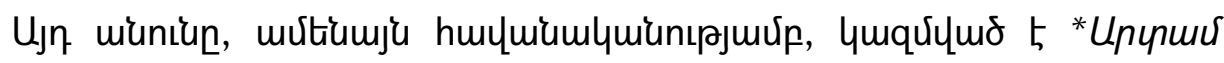

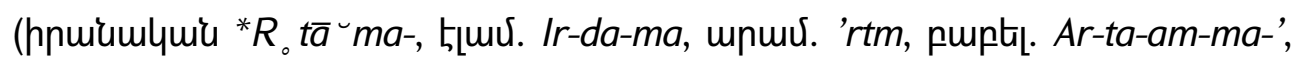

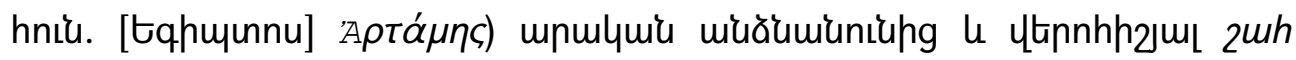




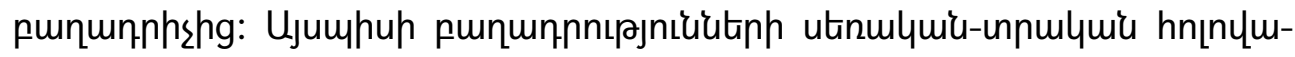

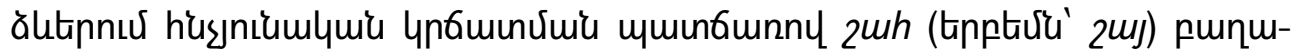

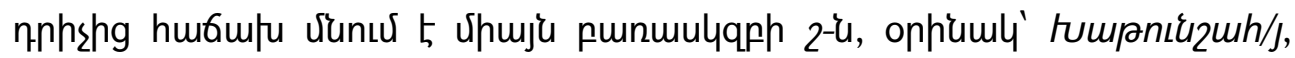

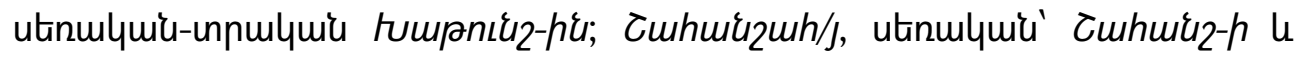

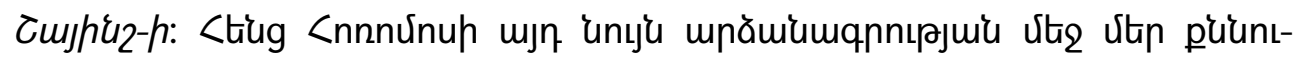

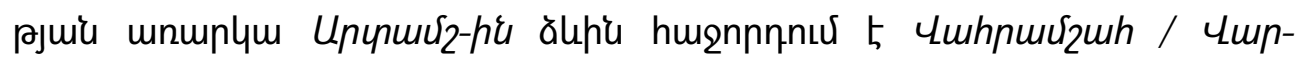

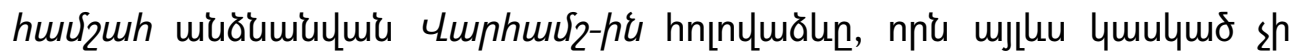

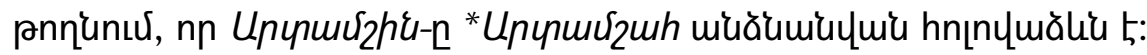

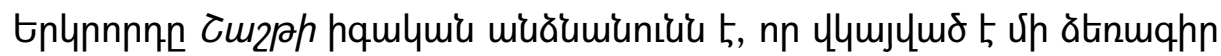

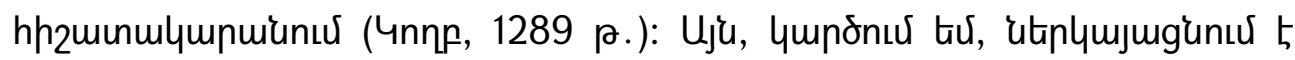

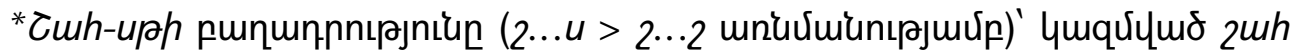

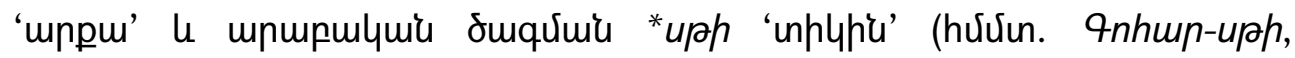

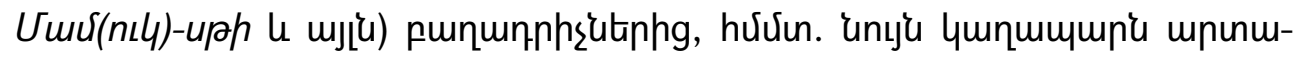

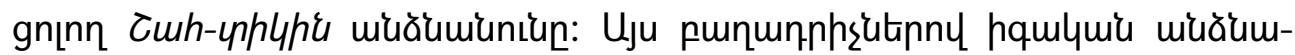

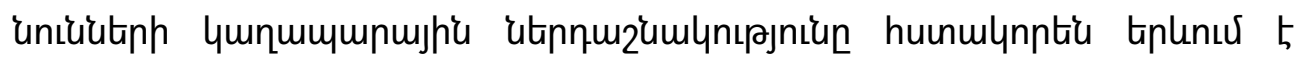
htinljü minnumulynu:

\begin{tabular}{|c|c|c|}
\hline & + yhyh\& & + uph 'unhlyhu' \\
\hline qnhwp + & 9nhwn-чhцhध & Gohar-u(h)ph \\
\hline vuvu(u) + & Uuvou-цhцhध & Uuvर(nıl)-uph \\
\hline $2 w h+$ & zuh-чphlhध & ${ }^{*} \widetilde{Z} w h-u p h$ \\
\hline
\end{tabular}

